DOI

ARTICULO ORIGINAL

Lorena Sainz* ORCID:0000-0002-5030-0699

Ana Regueira* ORCID: 0000-0002-7058-6612

Fernanda Ratto* ORCID: 0000-0002-2202-9010

Alvaro Munúa* ORCID: 0000-0001-7681-0657

Damián Da Luz* ORCID: 0000-0002-8201-9041

Gonzalo Aizpún* ORCID: 0000-0002-3343-9349

*Estudiante de Medicina. Facultad de Medicina. UdelaR

Gabriel Perez Pezzani Psicólogo clínico. UdelaR.

Federico Preve

Neurólogo. Asistente del Instituto de Neurología. Hospital de Clínicas UdelaR.

Andrés Lescano

Neuropsicólogo. Asistente de la Clínica de Neuropsicología. Hospita de Clínicas.

Federico Salle Neurocirujano. Asistente del Servicio de Neurocirugía. Hospital de

Clínicas.

Rodrigo Moragues ORCID: 0000-0002-3739-183X Neurocirujano. Profesor Adjunto de Servicio de Neurocirugía. Hospital de Clínicas.

Gastón Duarte* ORCID: 0000-0002-1559-7677

Lorena Tambasco** ORCID: 0000-0003-3258-527X

**Residente de Anestesia. Servicio de Anestesiología. Hospital de Clínicas. UdelaR.

Ronnie Henderson Anestesista. Asistente del Servicio de Anestesiología. Hospital de Clínicas. UdelaR.

Fernando Martínez ORCID: 0000-0002-6916-4738 Neurocirujano. Profesor Agregado del Servicio de Neurocirugía Hospital de Clínicas.

\section{Experiencia emocional percibida por pacientes sometidos a craneotomía con despertar intraoperatorio.}

\author{
Emotional experience perceived by patients undergoing craniotomy with \\ intraoperative awakening. \\ Experiência emocional percebida por pacientes submetidos à craniotomia \\ com despertar intraoperatório.
}

Resumen: Introducción: la craneotomía vigil para el tratamiento de tumores cerebrales en áreas elocuentes es una técnica que se realiza en forma cada vez más frecuente. Sin embargo, muy poca literatura se ha publicado en referencia a cómo percibe el paciente esta experiencia que podría parecer estresante. El objetivo de este trabajo es describir la experiencia emocional de los pacientes sometidos a despertar intraoperatorio para el tratamiento de los gliomas. Materiales y Métodos: Se realizó una revisión retrospectiva de 16 pacientes operados entre enero de 2015 y octubre de 2017, a los cuales se les efectuó una craneotomía con despertar intraoperatorio. Se identificaron 6 pacientes que cumplieron con los criterios de inclusión. Posteriormente a la cirugía se realizó, en una primera instancia, encuestas estandarizadas en donde se evaluó: datos sociodemográficos, nivel de ansiedad durante el despertar intraoperatorio, miedo a la anestesia y nivel de información de la enfermedad. En una segunda instancia, se le realizó una entrevista subjetiva por parte de un Licenciado en Psicología. Resultados: De los datos sociodemográficos obtuvimos que el $67 \%$ son hombres, y que la media de edad era de 30,66 años. En cuanto a la ansiedad observamos que el $60 \%$ de los pacientes tenían un nivel de ansiedad bajo, el $40 \%$ un nivel de ansiedad medio, y destacamos que ningún paciente tuvo un nivel de ansiedad alto. Con respecto al miedo a la anestesia y acto quirúrgico el $67 \%$ no tuvo miedo, mientras que el $37 \%$ restante afirman tenerlo, y los motivos más frecuentes para ello fueron: a morir, y a quedar con secuelas. En cuanto al nivel de información que fue proporcionada por el médico, en un $17 \%$ fue excelente, $33 \%$ muy completa, el $33 \%$ indicaron que la información fue suficiente y el $17 \%$ restante indica que la misma fue escasa. Conclusión: Destacamos que la cirugía con despertar intraoperatorio es bien tolerada. De las lecciones prácticas aprendidas enfatizamos la importancia de la entrevista preoperatoria en donde se explican con detalle el objetivo del procedimiento y cuáles son los pasos a seguir, para de esta manera maximizar la cooperación.

Palabras Clave: Craneotomía vigil, Ansiedad, Aspectos psicológicos, Tumor cerebral.

Abstract: Introduction: awake craniotomy for brain tumors surgery is a technique that is performed for tumors located on eloquent areas. However, a few articles has been published in reference to how the patient perceives this experience. Our objective is to describe the emotional experience of patients undergoing intraoperative awakening for the treatment of gliomas. Materials and Methods: retrospective review of 16 patients operated between January 2015 and October 2017, who underwent awake craniotomy. Six patients who met the inclusion criteria were identified. After the surgery, standardized surveys were carried out, in the first instance, where the following were evaluated: sociodemographic data, level of anxiety during intraoperative awakening, fear of anesthesia and level of information about the disease. In a second instance, a subjective interview was conducted by a Psychologist. Results: there were 4 mans and 2 womens with average age was 30.66 years. Regarding anxiety, we observed that $60 \%$ of patients had a low anxiety level, $40 \%$ had a medium level of anxiety, and we emphasized that no patient had a high level of anxiety. Regarding the fear of anesthesia and surgery, $67 \%$ were not afraid, while the remaining $37 \%$ claim to have fear of death or sequelae. Regarding the level of information 
that was provided by the doctor, $17 \%$ was excellent, $33 \%$ very complete, $33 \%$ indicated that the information was sufficient and the remaining $17 \%$ indicated that it was scarce. Conclusion: We emphasize that surgery with intraoperative awakening is well tolerated. From the practical lessons learned we emphasize the importance of the preoperative interview where the objective of the procedure is explained in detail and which are the steps to follow, in order to maximize cooperation.

Key words: Awake craniotomy, Anxiety, Brain tumor, Psychological aspects.

Resumo: Introdução: a craniotomia de vigília para o tratamento de tumores cerebrais em áreas eloqüentes é uma técnica cada vez mais realizada. No entanto, muito pouca literatura foi publicada em referência a como o paciente percebe essa experiência que pode parecer estressante. O objetivo deste trabalho é descrever a experiência emocional de pacientes submetidos ao despertar intraoperatório para o tratamento de gliomas. Materiais e Métodos: Revisão retrospectiva de 16 pacientes operados entre janeiro de 2015 e outubro de 2017, submetidos à craniotomia com despertar intraoperatório. Foram identificados seis pacientes que preencheram os critérios de inclusão. Após a cirurgia, em primeira instância, foram realizados inquéritos padronizados, nos quais foram avaliados: dados sociodemográficos, nível de ansiedade durante o despertar intraoperatório, medo da anestesia e nível de informação sobre a doença. Em um segundo momento, uma entrevista subjetiva foi conduzida por um Bacharel em Psicologia. Resultados: A partir dos dados sociodemográficos, obtivemos que $67 \%$ são homens e a idade média foi de 30,66 anos. Em relação à ansiedade, observamos que $60 \%$ dos pacientes apresentavam baixo nível de ansiedade, 40\% apresentavam nível médio de ansiedade e enfatizamos que nenhum paciente apresentava alto nível de ansiedade. Com relação ao medo da anestesia e da cirurgia, $67 \%$ não temeram, enquanto os $37 \%$ restantes afirmaram tê-lo, e os motivos mais frequentes foram: morrer e ficar com seqüelas. Em relação ao nível de informação que foi fornecido pelo médico, 17\% foi excelente, $33 \%$ muito completo, $33 \%$ indicaram que a informação era suficiente e os $17 \%$ restantes indicaram que esta era escassa. Conclusão: enfatizamos que a cirurgia com despertar intraoperatório é bem tolerada. A partir das lições práticas aprendidas, enfatizamos a importância da entrevista pré-operatória, onde o objetivo do procedimento é explicado em detalhes e quais são os passos a seguir, a fim de maximizar a cooperação.

Palavras-chave: Vigilância Craniotomia, Ansiedade, Aspectos Psicológicos, Tumor Cerebral.

Recibido: 10/10/2018 - Aceptado: 28/01/2019

Servicio de Neurocirugía. Hospital de Clínicas. "Dr. Manuel Quintela". Piso 2. Facultad de Medicina. Universidad de la República (UdelaR). Montevideo, Uruguay. Correspondencia: E-mail: fermartneuro@gmai.com 


\section{Introducción}

La neurocirugía ha tenido enormes avances en los últimos 20 años, que han ayudado a abatir la mortalidad y la morbilidad de los procedimientos quirúrgicos ${ }^{(1-3)}$. Mejorar los índices de morbimortalidad es fundamental para la seguridad y sobrevida digna del paciente.

Dentro de los avances destacables está el uso cada vez mas difundido de la craneotomía con despertar operatorio, también llamada "craneotomía vigil". Esto consiste en despertar al paciente durante la cirugía para monitorizar áreas elocuentes cerebrales (lenguaje, visión, motricidad) así como las vías de sustancia blanca que conectan estas áreas entre sí (1-6).

Como es de esperar, no todos los pacientes pueden ser sometidos a una craneotomía vigil, y la técnica se selecciona en base a múltiples aspectos, entre los que están: el tipo de lesión, su ubicación con respecto a las áreas y tractos de sustancia blanca cerebral e incluso, lo que manifieste el paciente durante la entrevista pre-quirúrgica ${ }^{(5,7)}$.

Un tema poco abordado en la literatura: es como vive el paciente la experiencia de ser despertado durante una cirugía con el cráneo abierto?

Nuestro objetivo para este trabajo fue evaluar las emociones y miedos que puedan haber sentido un grupo de pacientes sometidos a craneotomía vigil.

\section{Material y métodos}

Se llevó a cabo un estudio observacional descriptivo, de carácter retrospectivo en un grupo de pacientes operados por el mismo grupo de cirujanos (FM, FS, RM) entre setiembre de 2014 y octubre de 2017. En ese lapso, el grupo de los autores realizó 16 cirugías con despertar operatorio.

\section{D) Selección de pacientes para craneotomía vigil y técnica anestésico- quirúrgica}

En todos los casos, los pacientes fueron entrevistados por un integrante del equipo quirúrgico quien sugirió realizar el procedimiento con despertar operatorio en base a la topografía de la lesión y su relación con estructuras elocuentes (áreas corticales o tractos de sustancia blanca). Posteriormente el paciente es visto por uno de los neurólogos (FP, AL) para identificar la comprensión de los tests intraoperatorios. De la evaluación de ambos integrantes del equipo surge si el paciente está apto o no para ser sometido a craneotomía vigil.

Seleccionado el paciente para el procedimiento, el mismo se realiza en nuestro centro de la siguiente forma: 1) anestesia intravenosa total con propofol y remifentanilo con ventilación por mascara laríngea, 2) anestesia local en puntos del cabezal, sitio de incisión, aferencias nerviosas del colgajo cutáneo y del músculo temporal, 3) confección del colgajo cutáneo y óseo, 4) infiltración de la duramadre en línea de incisión con lidocaína al 1\%, 5) se procede al despertar y retiro de mascara laríngea antes de abrir la duramadre, 6) ecografía intraoperatoria para ubicación y valoración de la extensión de la lesión, 7) mapeo cortical y de sustancia blanca con resección de la lesión. En todo momento el neurólogo hace testeo de lenguaje y motricidad. Durante esta parte de la cirugía el paciente solo recibe dexmedetomidina y analgésicos si son necesarios, 8) resecada la lesión y realizada la hemostasis se procede a colocar nuevamente la mascara laríngea, para el cierre bajo anestesia general. Durante todo el procedimiento se pregunta al paciente si está cómodo o siente dolorademás de hacer los testeos motor y dellenguaje.

\section{E) Criterios de inclusión y exclusión}

Criterios de inclusión: edad entre 15 y 64 años (Criterios usados por el Comité de cirugía con despertar operatorio de la Sociedad Japonesa de Neurocirugía), diagnóstico de glioma de bajo grado sometidos a cirugía con despertar intraoperatorio y operados por alguno de los cirujanos del equipo (FM, RM, FS), utilizando la misma técnica.

Criterios de exclusión: no se incluyeron pacientes que sufrieron incidentes o alteraciones de la conciencia durante el procedimiento. Se excluyeron asimismo pacientes con trastornos persistentes en el lenguaje y pacientes que no puedan firmar el consentimiento informado. 


\section{F) Procedimiento}

Consistió en una entrevista que se desarrolló en dos etapas. En la primera se realizó una encuesta estandarizada y la segunda constó de una entrevista subjetiva con un Licenciado en Psicología (GPP).

Las variables consideradas mediante las encuestas estandarizadas fueron: datos patronímicos (edad, género, nivel educativo y estado civil), nivel de ansiedad durante el despertar intraoperatorio (mediante la Escala State-traite anxietyinventory (STAI), miedo al acto quirúrgico y a la anestesia y finalmente, el nivel de información recibido previo a la cirugía.

En la segunda etapa de las entrevistas, el Licenciado en Psicología identificó rasgos de personalidad haciendo preguntas dirigidas de respuesta abierta.

El protocolo de investigación fue aprobado por el Comité de Ética del Hospital de Clínicas y se obtuvo el consentimiento informado de los pacientes.

\section{Resultados}

De los 16 pacientes operados 6 que cumplieron con los criterios de inclusión y pudieron ser ubicados para realizar una entrevista presencial.

Se trato de 4 hombres y dos mujeres de entre 16 y 39 años (media 30.6), cuyo nivel de instrucción fue: primaria incompleta (1), primaria completa (1), secundaria incompleta (3) y secundaria completa (1).

En cuanto a la evaluación del estado de ansiedad (mediante la escala STAI), un paciente manifestó no recordar nada de lo ocurrido durante la cirugía y por lo tanto, no podemos valorar adecuadamente su respuesta. Los otro cinco tuvieron valores de STAI entre 20 y 41 (media 27). Para la escala STAI, los puntajes mayores a 45 muestran un alto grado de ansiedad, entre 30 y 44 un grado medio (dos pacientes) y menos de 30 puntos cataloga para un bajo grado de ansiedad ( 3 pacientes). Es decir, un paciente no parece haber vivido la experiencia como traumática pero al no dar puntaje no podemos valorar adecuadamente su respuesta, pero de los 5 que si pudimos evaluar, ninguno tuvo durante el procedimiento un grado alto de ansiedad y la mayoría tuvieron un bajo grado de ansiedad. Al correlacionar el puntaje de STAI con la duración de la cirugía, observamos que, si bien el número de la muestra es bajo, hay una correlación entre menor tiempo quirúrgico y menor grado de ansiedad. Los pacientes que tuvieron grados de ansiedad bajos tuvieron tiempos de despertar de una hora o inferiores.

Sobre el miedo a la anestesia y la cirugía, dicha variable se evaluó mediante las siguientes preguntas: 1) ¿tuvo miedo a la anestesia?, 2) ¿motivos del miedo?, 3) si no tuvo miedo se le interrogó sobre cuales fueron los motivos que identificó para no sentir miedo, 4) ¿tuvo visita pre anestésica? 5) argumentos para tener confianza en el equipo quirúrgico.

El $67 \%$ de los pacientes no tuvo miedo a la anestesia, mientras que el 33\% restante sí lo tuvo. Consultados por el motivo del miedo, las opciones marcadas con más frecuencia fueron el miedo a morir o a quedar con secuelas.

Respecto a los argumentos más importantes de los pacientes que no tuvieron miedo, el $50 \%$ fue debido a que ya había recibido anestesia general; un $25 \%$ porque quería operarse, mientras que el $25 \%$ restante atribuyó su confianza a la explicación previa de cómo sería el procedimiento.

En relación a la interrogante si tuvieron visita pre anestésica, el $83 \%$ la tuvo, mientras que el $17 \%$ restante no.

Consultados por los argumentos para tener confianza en el equipo quirúrgico, el $83 \%$ lo atribuyó tanto a la confianza en el médico como al trato humano, mientras que un $17 \%$ solo lo relaciona a la confianza en el médico.

Interrogamos a los pacientes sobre el conocimiento del procedimiento y la enfermedad mediante las siguientes preguntas: 1) ¿conoce la enfermedad que padece?, 2) ¿conoce los resultados de las pruebas que le han hecho?, 3) ¿Ocurrió algo inesperado por usted durante la cirugía? 4) ¿cómo valoraría la información que la ha proporcionado su médico?

Sobre el conocimiento de su enfermedad, 3 pacientes respondieron que la conocían poco o nada y 3 que la conocían bastante o mucho. 
En relación al conocimiento de los resultados de las pruebas que le han hecho, 2 manifestaron no saber nada y 4 que conocían bastante o un poco.

En cuanto a la información proporcionada 5 pacientes valoraron la información como muy completa o suficiente y solo uno la valoró como escasa.

En cuanto a si sucedió algo en el intra-operatorio, 67\% indicaron que se encontraron con situaciones que no esperaban. Los motivos expresados por estos pacientes se profundizaron en la entrevista con el psicólogo.

Los datos obtenidos en la entrevista subjetiva son más difíciles de estandarizar y tabular, por lo tanto se ordenaran por categorías ya que los pacientes entrevistados presentan algunos rasgos comunes:

1) La especificidad del proceso operatorio y el cambio de paradigma en el tratamiento genera actitudes positivas y un interés estimulante. Esto lo manifiestan el paciente, el grupo de estudiantes y los neurocirujanos. La cooperación del paciente obliga a tomar en cuenta su subjetividad e invita a una sinergia de trabajo con una apertura a la pluri-disciplinariedad.

2) Algunos de los pacientes parecen un poco "sumisos" a la autoridad médica, más allá del rol de colaboradores que se le otorga. "Entregarse totalmente" al cirujano que los va a operar, puede ser debido a un mecanismo de defensa. En algunas oportunidades firman el consentimiento informado sin leerlo. Esa aceptación del sujeto puede interpretarse como una adaptación necesaria que permite procesar el sentimiento de miedo con la posibilidad de muerte que acompaña el imaginario de ir al block quirúrgico. Para ellos es mejor tener confianza en el que sabe (el neurocirujano) y darle total confianza, sin necesidad de controlar.

3) Podemos observar que la memoria, los recuerdos del despertar intra-operatorio es variable: algunos pacientes recuerdan colores, palabras, toda la fase del trabajo con el neuropsicólogo que le pide nombrar las imágenes en una pantalla. Otros se recuerdan del ambiente general, a veces reportan bromas que les hacían los cirujanos o que escucharon entre los profesionales como forma de relajar el ambiente. Se nombraron molestias que tenían que ver sobre todo con la postura.

4) Las consecuencias psicológicas en el paciente de la cirugía con despertar es también variable. Observamos que podía desencadenar episodios paranoides con sentimientos persecutorios referidos al despertar. Por ejemplo un paciente dice recordar un momento del despertar en el cual relata "me recuerdo estar rodeado de gente, tomando fotos, hablando, escucho risas" sumado a un momento difícil de su rehabilitación psicosocial ya que "me miran distinto desde que volví" (a su ambiente habitual). El día de la entrevista este paciente llora mucho y dice no reconocerse. Comparte su nostalgia del periodo anterior a la operación. En cambio otro paciente, también hombre, cambió totalmente su vida y "agradece" haber encontrado con la operación y ese tumor un límite a una vida enfocada en los aspectos superfluos.

5) Los pacientes son generalmente de nivel socio económico bajo y de formación básica. Ese aspecto no explicaría en sí que se sometan, se entreguen, más fácilmente a la autoridad médica.

6) Señalamos también que la mayoría de los pacientes que entrevistamos fueron acompañados por un tercero, sostén, mediador, traductor o porta voz. Sugiere cierta forma de dependencia afectiva y socio-cognitiva. Genera sin duda beneficios secundarios. Los pacientes que entrevistamos habían suspendido sus actividades laborales, lo que se acompañó de un sentimiento fuerte de inutilidad social.

\section{Discusión}

La cirugía con despertar operatorio puede usarse en varios tipos de neurocirugías que incluyen: hematomas subdurales crónicos, epilepsia, malformaciones vasculares, tumores y trastornos del movimiento. No todas estas cirugías entran en la misma categoría por la complejidad y duración de cada tipo de procedimiento, pero si comparten algo en común: el individuo se despierta "con el cráneo abierto". Si bien para un neurocirujano realizar un abordaje a un tumor es habitual, no es tan frecuente hacerlo con el paciente despierto ${ }^{(1-7)}$.

Los autores de este reporte estamos convencidos de que las indicaciones son cada vez mas frecuentes, si queremos mejorar la morbilidad de los tumores ubicados cerca de áreas o tractos de sustancia blanca elocuentes. Según Duffau, "una planificación preoperatoria cuidadosa por parte de un equipo multidisciplinario, dedicado a un paciente informado, sigue siendo un 
requisito previo importante para una craneotomía despierta con éxito" (8). Esta frase jerarquiza varios aspectos a ser tenidos en cuenta: 1) paciente informado y 2) equipo multidisciplinario.

Nos parece fundamental que el paciente tenga claro como es todo el procedimiento para que lo viva de la forma menos traumática posible. A pesar de que en cada entrevista preoperatoria el cirujano esta más de media hora con cada paciente y que este es visto por el neurólogo y luego es visto nuevamente por el cirujano, un paciente de nuestra serie valoró la información que se le dio como escasa.

La esfera psicológica es un aspecto fundamental en el abordaje de todo paciente, un aspecto muchas veces subestimado, y a veces hasta desvalorado en la práctica quirúrgica. Paradójicamente, algunas de las complicaciones frecuentes que pueden sufrir los pacientes quirúrgicos (y más exactamente aquellos con tumores cerebrales) son los trastornos neuropsiquiátricos. Solo el anuncio del diagnóstico de un tumor cerebral genera un estrés psicológico enorme ${ }^{(7,9,10)}$. La experiencia de ser operado de un tumor cerebral despierto puede parecer traumática para los pacientes. Cabe destacar que el paciente solamente permanece despierto cuando se realiza la estimulación cortical y subcortical y la resección tumoral, con lo cual se evitan todas las molestias en relación con la apertura y el cierre de la craneotomía.

Una investigación realizada por la Universidad de Edimburgo evaluó la percepción del despertar intraoperatorio en pacientes con tumores cerebrales. La mayoría recordo varios aspectos del procedimiento, aunque un $20 \%$ no recordaban estar despiertos durante la cirugía a pesar de ser cooperativos ${ }^{(9)}$. Los pacientes reportaron: algunas molestias leves (20\%), miedo (15\%) o ansiedad (29\%). Sin embargo la mayoría sintió que lidiaron con las pruebas de buena manera ${ }^{(9)}$. En otra serie de pacientes, solo $24 \%$ manifestó algún grado de disconfort y $80 \%$ estaban conformes con la experiencia vivida ${ }^{(11)}$. Nuestra experiencia es similar: un paciente bien informado y consciente de que el despertar es para su beneficio es un "aliado" invalorable para este tipo de procedimientos.

Un estudio sobre 21 pacientes mostró que los mismos están concentrados en controlar sus emociones y que se sienten responsables del resultado oncológico ${ }^{(12)}$. Creemos que este aspecto es importantísimo: comprometer al paciente con el procedimiento y que comprenda que es parte del equipo ya que el éxito de la cirugía depende en parte de su colaboración. Por otra parte, esta colaboración se logra con un equipo comprometido y que es capaz de contener e informar adecuadamente al paciente sobre lo que ocurrirá dentro de la cirugía ${ }^{(13,14)}$.

Estos aspectos han sido estudiados también para otras cirugías con paciente despierto y permiten identificar que cosas generan estrés o ansiedad en los pacientes ${ }^{(15)}$. Hay varios factores identificados que generan o disminuyen la ansiedad u estrés intra-operatorio, por ejemplo, en general las mujeres sienten más ansiedad que los hombres ${ }^{\left({ }^{15}\right)}$. El dolor, la incomodidad, el uso de palabras como "bisturí" o palabras de difícil comprensión para el paciente parecen aumentar la ansiedad y el estrés. Por otro lado, el poner música agradable para el paciente antes y dentro del procedimiento, el uso de palabras suaves, la conversación trivial entre los integrantes del equipo y la buena comunicación están asociados a menores niveles de ansiedad y estrés (15). Un trabajo inglés sobre 3 pacientes, mostró que la presencia de un familiar en la sala quirúrgica puede mejorar los niveles de ansiedad ${ }^{(16)}$. Si bien esta estrategia puede ser utilizada, creemos que debe valorarse adecuadamente su uso.

Si bien parece lógico pensar que los aspectos sicológicos o de la personalidad pueden afectar el desempeño del paciente durante la cirugía y su aceptación de la misma, no hay muchas investigaciones publicadas sobre el tema. No obstante en varios estudios se ha investigado la percepción de los pacientes durante el despertar intra-operatorio ${ }^{(7,9-17)}$ Estos estudios demostraron que este tipo de cirugías generalmente son bien toleradas por los pacientes. Es más, a medida que pasa el tiempo los pacientes tienen mejor percepción del procedimiento. Por ejemplo, en un estudio sobre 21 pacientes, la mitad manifestó estar conforme con el procedimiento en tanto la otra mitad manifestó molestias menores o moderadas en el intra-operatorio (dolor, sed, posicionamiento inadecuado). Al mes, todos manifestaron que el procedimiento los dejó muy conformes ${ }^{(17)}$. Otro estudio sobre 105 pacientes mostró altos grados de conformidad, bajos grados de dolor o molestias intraoperatorias (media 2.1 en escala EVA) y bajos niveles de ansiedad $(2.6 / 10){ }^{(18)}$.

Un hallazgo relativamente constante es que pacientes jóvenes y de sexo femenino manifiestan mayores niveles de ansiedad y que el disconfort intraoperatorio se ve en relación con el cabezal de pinchos y la posición en la mesa operatoria ${ }^{(16-18)}$.

Es muy importante la entrevista preoperatoria en donde se explican con detalle el objetivo del procedimiento y de esta manera se logra preparar a los pacientes a fondo para reducir la 
ansiedad y maximizar la cooperación. Gracias a estas intervenciones simples antes de la cirugía los pacientes presentan en general una actitud muy positiva ${ }^{(13,19)}$.

Otro estudio demostró que la cirugía en algunos pacientes podría tener un efecto positivo en los trastornos de ansiedad reactiva ${ }^{\left({ }^{(19)}\right.}$. En el mismo se enfatiza la evaluación neuropsicológica y la rehabilitación postoperatoria. Para los pacientes es crucial que los mismos terapeutas, hagan un seguimiento regular a largo plazo para un mayor apoyo psicológico y de esta manera, mantener una mejor calidad de vida.

\section{Conclusiones}

La cirugía de tumores cerebrales con paciente despierto en general es bien tolerada.

Es necesario explicar al paciente como se desarrollará el procedimiento y sus eventuales complicaciones (crisis epilépticas) para evitar aumentar la ansiedad.

Es necesario el trabajo multidisciplinario y el constante apoyo a los pacientes antes, durante y después de la cirugía.

En nuestra experiencia con un bajo numero de pacientes, el nivel de ansiedad manejado fue entre bajo y medio y esto estuvo en relación con la duración del despertar.

La mayoría de los pacientes no tuvieron miedo al acto anestésico quirúrgico.

En lo que refiere a las perspectivas que nos aporta esta investigación destacamos que sería beneficioso realizar un estudio con mayor número de pacientes donde contemos con evaluaciones de ansiedad y depresión previas a la cirugía y posteriores a ésta para realizar grupos de comparación.

En investigaciones futuras sería interesante valorar el impacto psicosocial que tiene para el paciente tanto la enfermedad como la cirugía, incluyendo en estas evaluaciones entrevistas con miembros de la familia como fuente cerca y válida de información.

Finalmente podría ser beneficioso intentar llegar a un consenso en el equipo tratante a fin de crear un ambiente estandarizado y propicio donde se minimicen al máximo las incomodidades que puedan generarse en el paciente.

\section{Bibliografía}

1- Martínez F, Salle F, Moragues R, Bertullo G. Utilidad de la estimulación intra- operatoria en cirugía de procesos expansivos intracraneanos bajo anestesia general. 63.

Rev. Urug. med. Interna. 2017; 1:58-

2- Duffau H, ed. Difuselow grade gliomas in adults. London: Springer, 2013. Introduction,

Fromtheinhibition of dogmas to the concept of personalized management in diffuse low grade gliomas; p. 1-5.

3- Sawaya R, Hammoud M, Schoppa D, Hess KR, Wu SZ, Shi WM, et al. Neurosurgical outcomes in a modern series of 400 craniotomies for treatment of parenchymal tumors. Neurosurgery 1998;42(5):1044-1056.

4- Asthagiri AR, Pouratian N, Sherman J, Ahmed G, Shaffrey ME. Advances in brain tumor surgery. Neurol Clin. 2007;25: 975-1003.

5- Martinez F, Moragues R. Cirugía tumoral con despertar intraoperatorio: es posible en Uruguay? Opción Méd. 2016;64:4-8.

6- Duffau H, ed. Difuse low grade gliomas in adults. Springer, London, 2013. Surgery for diffuse low grade gliomas. Functional considerations; p. 375-400.

7- Ruis C, Wajer IH, Robe P, van Zandvoort M. Anxiety in the preoperative phase of awake brain tumor surgery. Clin Neurol Neurosurg 2017;157:7-10.

8- Duffau $\mathrm{H}$. The error of Broca: From the traditional localizationist concept to a connectomal anatomy of human brain. J Chem Neuroanat. 2018;89:73-81

9- Whittle IR, Midgley S, Georges H, Pringle A-M, Taylor R. Patient perceptions of "awake" brain tumour surgery. Acta Neuro chir (Wien) 2005;147(3):275-277. 
10- Howie E, Bambrough J, Karabatsou K, Fox JR. Patiente xperiences of awake craniotomy: an Interpretative Phenomenological Analysis. J Health Psychol 2016; 21(11):2612-2623.

11- Wahab SS, Grundy PL, Weidmann C. Patient experience and satisfaction with awake craniotomy for brain tumours. Br J Neurosurg. 2011;25(5):606-613.

12- Palese A, Skrap M, Fachin M, Visioli S, Zannini L. The Experience of Patients Undergoing Awake Craniotomy: in the patients own words. A quialitative study. Cancer Nurs 2008;31(2):166-172.

13- Mitchell M. Conscious surgery: influence of the environment on patient anxiety. J Adv Nurs 2008;64(3):261-71.

14- Mitchell M. Patientanxiety and conscious surgery. J Perioper Pract 2009;19(6):168-173.

15- Caddick J, Jawad S, Southern S, Majumder S. The power of words: sources of anxiety in patient sunder going local anaesthetic plastic surgery. Ann R Coll Surg Engl 2012;94(2): 94-98.

16- Whittle IR, Lim JX. Over coming fear and anxiety during awake resection of brain tumours: family support can be pivotal to a successful outcome.Br J Neuro Surg 2013;27(1):117-118.

17- Danks RA, Rogers M, Aglio LS, Gugino LD, Black PM. Patient tolerance of craniotomy performed with the patient under local anesthesia and monitored conscious sedation. Neurosurgery. 1998;42(1):28-3436 .

18- Beez T, Boge K, Wager M, Whittle I, Fontaine D, Spena G, et al. Tolerance of awake surgery for glioma: a prospective European Low Grade Glioma Network multicenter study. Acta Neuro chir 2013;155(7):1301-1308.

19- Khu KJ, Doglietto F, Radovanovic I, Taleb F, Mendelsohn D, Zadeh G, et al. Patients' perceptions of awake and outpatient craniotomy for brain tumor: a qualitative study. J Neuro surg 2010;112(5):10561060.

\section{Aporte cada autor al trabajo}

Lorena Sainz: Recolección de datos y entrevistas

Ana Regueira: Recolección de datos y entrevistas

Fernanda Ratto: Recolección de datos y entrevistas

Alvaro Munúa: Recolección de datos y entrevista

Damián Da Luz: Recolección de datos y entrevista

Gonzalo Aizpún: Recolección de datos y entrevista

Gabriel Perez Pezzani: Recolección de datos, entrevista, planificación de entrevista, selección de escalas y análisis de datos.

Federico Preve: Revisión del manuscrito, testeo intraoperatorio.

Andrés Lescano: Revision del manuscrito, testeo intraoperatorio.

Federico Salle: Planificación del artículo, confección y revisión del manuscrito selección de escalas, análisis de datos.

Rodrigo Moragues: Confección y revisión del manuscrito.

Gastón Duarte: Revisión del manuscrito, planificación de protocolo anestésico.

Lorena Tambasco: Revisión del manuscrito, planificación de protocolo anestésico.

Ronnie Henderson: Revisión del manuscrito, planificación de protocolo anestésico.

Fernando Martínez: Planificación del artículo, confección y revisión del artículo, análisis e interpretación de datos. 